\title{
On Receiver Design for Uplink Low Density Signature OFDM (LDS-OFDM)
}

\author{
Razieh Razavi, Student Member, IEEE, Mohammed AL-Imari, Student Member, IEEE, \\ Muhammad Ali Imran, Member, IEEE, Reza Hoshyar, Member, IEEE, and Dageng Chen, Member, IEEE
}

\begin{abstract}
Low density signature orthogonal frequency division multiplexing (LDS-OFDM) is an uplink multi-carrier multiple access scheme that uses low density signatures (LDS) for spreading the symbols in the frequency domain. In this paper, we introduce an effective receiver for the LDS-OFDM scheme. We propose a framework to analyze and design this iterative receiver using extrinsic information transfer (EXIT) charts. Furthermore, a turbo multi-user detector/decoder (MUDD) is proposed for the LDS-OFDM receiver. We show how the turbo MUDD is tuned using EXIT charts analysis. By tuning the turbo-style processing, the turbo MUDD can approach the performance of optimum MUDD with a smaller number of inner iterations. Using the suggested design guidelines in this paper, we show that the proposed structure brings about $2.3 \mathrm{~dB}$ performance improvement at a bit error rate (BER) equal to $10^{-5}$ over conventional LDS-OFDM while keeping the complexity affordable. Simulations for different scenarios also show that the LDS-OFDM outperforms similar well-known multiple access techniques such as multi-carrier code division multiple access (MC-CDMA) and group-orthogonal MCCDMA.
\end{abstract}

Index Terms-Multi access communication, Iterative methods, Code division multi access, Multi-user channels.

\section{INTRODUCTION}

$\mathbf{M}$ ULTI-carrier code division multiple access (MCCDMA) is considered to be a suitable approach to cope with challenging service demands due to its ability of exploiting both time and frequency resources [1]-[4]. In the uplink channel using MC-CDMA, non-orthogonality of received effective signatures causes multi-user interference (MUI). Multi-user detection (MUD) can be used to moderate the detrimental effects of MUI. However, implementation of optimum MUDs is not practical due to their prohibitively high computational complexity. Even for a moderate number of interfering users, the complexity grows exponentially with the number of users [5]. In order to reduce the complexity of MUD for MC-CDMA systems, group-orthogonal MC-CDMA (GO-MC-CDMA) [6] has been proposed. It has been shown that by dividing users into subgroups, GO-MC-CDMA is able to achieve a performance very close to the single-user bound [6] while keeping the complexity affordable.

In order to achieve a further reduction in the complexity of MUD for uplink MC-CDMA, we have recently proposed low density signature (LDS) structures for MC-CDMA systems

R. Razavi, M. AL-Imari and M. A. Imran are with the Centre for Communications Systems Research, University of Surrey, Guildford, Surrey, GU2 7XH, UK, (e-mail: R.Razavi, M.Al-imari, M.Imran @ surrey.ac.uk).

R. Hoshyar is with Texas Instruments, California, USA.

D. Chen is with Huawei Technologies Co. Ltd, No.2222, Xin JinQiao Road, Pudong, Shanghai, 201206, P.R.China. known as LDS-OFDM [7]. In LDS-OFDM systems, because of their low density signature structures, each data symbol is only spread over a limited number of chips (effective processing gain). Then, each user's generated chip is transmitted over an orthogonal sub-carrier. Furthermore, each sub-carrier is only used by a limited number of data symbols that may possibly belong to different users. Therefore each user, transmitting on a given sub-carrier, will experience interference from only a small number of other data symbols. In other words, LDSOFDM is a special case of the MC-CDMA system where its spreading sequences have low density. In theory, low density signatures were introduced in [8] for downlink multi-carrier systems. Since the LDS-OFDM system is proposed for uplink multi-carrier systems, the design of the receiver is different from the one proposed in [8], because in uplink we have a single centralised receiver. Designing this receiver to have minimal or affordable complexity while being able to achieve the single user performance bound is a challenge. This paper aims to address this challenge by proposing a suitable design and analysis tool to obtain an efficient detection/decoding algorithm. The contributions of this paper are as follows:

1) An efficient implementation of the message-passing algorithm (MPA) [9] to approximate the maximum $a$ posteriori (MAP) detection for the LDS structure is presented. This algorithm is able to reduce the complexity of the receiver compared to the existing state-of-theart multiple access systems. This technique calculates the extrinsic information for each sub-carrier by using the MAP-based detections: Log-MAP with brute-force searching among possible combinations of symbols.

2) The iterative detector is analysed using extrinsic information transfer (EXIT) charts. Furthermore, design guidelines are provided for improving the LDS structure.

3) A tuned turbo multi-user detector/decoder (MUDD) for LDS-OFDM is proposed. The proposed turbo receiver comprises two basic components: an LDS multi-user symbol detector and a collection of users' decoders. Using the EXIT chart analysis we show how to tune the receiver in a way that the turbo MUDD can approach its final performance using a smaller number of inner iterations. In [10], we have already shown a primary study on LDS-OFDM with turbo iterations to demonstrate the effect of various loads, and here we extend this work further by showing how the turbo receiver is tuned using a novel technique.

4) Finally, using design guidelines extracted from the EXIT 


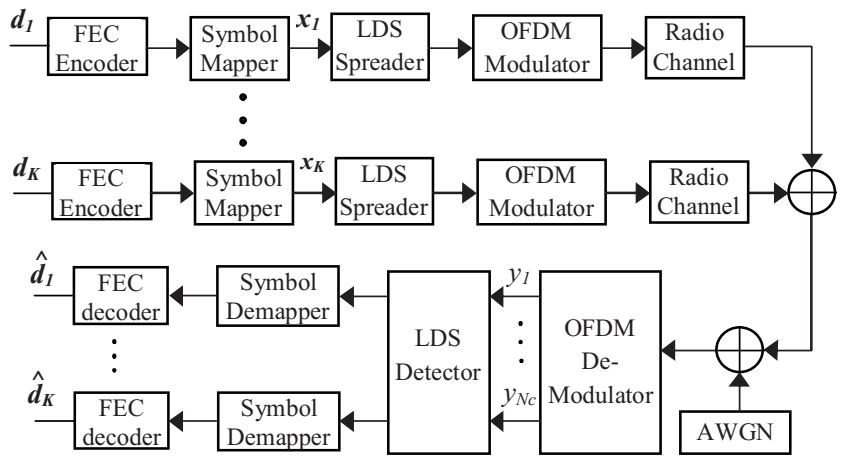

Fig. 1. LDS-OFDM block diagram.

chart analysis of the receiver, we provide the BER results to show why LDS-OFDM is a promising technique in comparison with well-known multiple access techniques with similar principles.

The rest of this paper is organized as follows. Section II presents the structure of LDS-OFDM and its iterative receiver. In section III, the design guidelines for an LDSOFDM receiver is extracted using EXIT chart analysis. The analysis of turbo MUDD for LDS-OFDM is presented in section IV, and then the simulation results and comparison with similar techniques are both given in section V. In section VI, different aspects of the LDS-OFDM system are compared with those of other multiple access techniques. Finally, section VII is devoted to concluding remarks.

\section{LDS-OFDM SYSTEM MODEL}

Consider a multiple access channel (MAC), corresponding to the uplink communications from multiple users to a single base station in a practical system. The block diagram of LDSOFDM for an uplink system is shown in Fig. 1. It can be noted that the main blocks are similar to an MC-CDMA system. Similar to an MC-CDMA spreading process, we multiply the modulated symbol with a spreading signature (a random sequence of chips). However, in the LDS-OFDM case, the main difference is that the spreading signature has a low density (a large number of chips in the sequence are equated to zero). In this section, we first explain the system model of LDS-OFDM, which is similar to the one published in [10]. Then the iterative receiver for this system is described. It is assumed that the LDS-OFDM system has $K$ users with user indices $k=1, \ldots, K$. Let $\mathbb{X}_{k}$ be the constellation alphabet, from which the transmitted symbol for user $k$ will take its value. Thus, the modulated symbol for user $k$ is formed by mapping a sequence of independent information bits $\mathbf{b}_{k} \in \mathbb{F}_{2}^{q_{k}}$ to $\mathbb{X}_{k}$, and can be represented by the function $\phi_{k}: \mathbb{F}_{2}^{q_{k}} \rightarrow \mathbb{X}_{k}$, where $q_{k}$ is the number of information bits per symbol for user $k$. Without loss of generality, all users are assumed to take their symbols from the same constellation alphabet, i.e., $\mathbb{X}=\mathbb{X}_{k}, \forall k=1, \ldots, K$. In addition, we assume that the different users have identical number of data symbols, $M$.

Introducing $N_{c}$ as the number of total chips, the spreading matrix for user $k$ will be $\mathbf{S}_{k}=\left[\mathbf{s}_{k, 1}, \ldots, \mathbf{s}_{k, M}\right] \in \mathbb{C}^{N_{c} \times M}$, where matrix $\mathbf{S}_{k}$ has only $d_{v}$ (effective spreading factor) nonzero values on each column. Let us define $\mathbf{S}=\left[\mathbf{S}_{1}, \ldots, \mathbf{S}_{K}\right] \in$ $\mathbb{C}^{N_{c} \times M K}$ as the low density signature matrix of the multiuser system. The norm of each signature vector is unity. Furthermore, let variable $d_{c}$ be the number of symbols that interfere with each other at each chip. We define $\mathbf{A}=$ $\operatorname{diag}\left(A_{1}, \ldots, A_{K}\right)$ as the transmit power gain of users and $\mathbf{G}_{k}=\operatorname{diag}\left(g_{k, 1}, \ldots, g_{k, N_{c}}\right)$ as the corresponding channel gain for the $k^{t h}$ user.

In LDS-OFDM, each user's generated chip will be transmitted over an orthogonal sub-carrier. Therefore, the received spreading sequence for data symbol $m \in\{1, \ldots, M\}$ of user $k$ can be represented by $\mathbf{h}_{k, m}=A_{k} \mathbf{G}_{k} \mathbf{s}_{k, m}$. To be more specific, the received signature gain at chip $n$ of data symbol $m$ of user $k$ will be $h_{k, m}^{n}=A_{k} g_{k, n} s_{k, m}^{n}$. Let $\mathcal{J}_{n}=\left\{(k, m): s_{k, m}^{n} \neq 0\right\}$ be the set of data symbols (which may belong to different users) that interfere on chip $n$. Also, let $\mathcal{E}_{k, m}=\left\{n: s_{k, m}^{n} \neq 0\right\}$ be the set of different sub-carriers that the $m^{\text {th }}$ symbol of user $k$ is spread on.

For an uplink MC-CDMA system, the received signal at sub-carrier index $n$ is written as

$$
y_{n}=\sum_{k=1}^{K} \sum_{m=1}^{M} h_{k, m}^{n} x_{k, m}+v_{n},
$$

where $v_{n}$ is the AWGN for sub-carrier $n$ and $x_{k, m}$ is the $m^{\text {th }}$ data symbol of user $k$. Thus, considering that in LDSOFDM system, the signature has a limited number of non-zero positions, we can express the received signal at the $n^{\text {th }}$ chip (sub-carrier) as follows:

$$
y_{n}=\sum_{(k, m) \in \mathcal{J}_{n}} h_{k, m}^{n} x_{k, m}+v_{n} .
$$

At the receiver side, after OFDM demodulation, the signal is sent to a near-optimum MUD based on MPA. An LDS system, with $K$ users and $N_{c}$ sub-carriers, is represented by the factor graph $\mathcal{G}(\mathcal{U}, \mathcal{C})$ where $\mathcal{C}$ and $\mathcal{U}$ are the sets of function nodes and variable nodes, respectively. Each chip is represented by a function node $c \in \mathcal{C}$ and each user-symbol is represented by a variable node $u \in \mathcal{U}$. The connections between the received sub-carrier and its related users are shown by edges. Let $e_{n}\left(x_{k, m}\right)$ be the edge that connects function node $n$ to variable node $m$ of user $k$. Because this receiver is iterative and operates at the chip-level, the resultant technique is termed the chip-level iterated (CLi) MUD. Because of small number of interferers in each sub-carrier, applying maximum a posteriori based chip-level iterated (MAP-CLi) MUD is feasible for LDS-OFDM. The design of this receiver is based on MPA, which requires iterative exchange of messages between the function and the variable nodes.

The CLi MUD technique can be explained as follows. Let $L_{n, \rightarrow}^{j}\left(x_{k, m}\right)$ be the message sent along the edge $e_{n}\left(x_{k, m}\right)$, at $j^{t h}$ iteration, from variable node $u_{k, m}$ to function node $c_{n}$. Similarly, the message sent from the function node to the variable node is given by $L_{n, \leftarrow}^{j}\left(x_{k, m}\right)$. Assuming there are no a priori probabilities available, the initial messages $(j=0)$ are set to zeros: $L_{n, \rightarrow}^{0}\left(x_{k, m}\right)=0, \forall k, m, \forall n$. The messages 
are updated using the following rules

$$
\begin{gathered}
L_{n, \rightarrow}^{j}\left(x_{k, m}\right)=\sum_{l \in \mathcal{E}_{k, m} \backslash n} L_{l, \leftarrow}^{j-1}\left(x_{k, m}\right), \\
L_{n, \leftarrow}^{j}\left(x_{k, m}\right) \propto \\
f\left(x_{k, m} \mid y_{n}, L_{n, \rightarrow}^{j}\left(x_{k^{\prime}, m^{\prime}}\right), \forall\left(k^{\prime}, m^{\prime}\right) \in \mathcal{J}_{n} \backslash(k, m)\right) .
\end{gathered}
$$

It can easily be seen from (3) and (4), that all messages are updated by the extrinsic information. In order to approximate the optimum MAP detector, the right hand side of (4) represents marginalization function, which is based on (2), and can be written as

$$
\begin{gathered}
f\left(x_{k, m} \mid y_{n}, L_{n, \rightarrow}^{j}\left(x_{k^{\prime}, m^{\prime}}\right), \forall\left(k^{\prime}, m^{\prime}\right) \in \mathcal{J}_{n} \backslash(k, m)\right)= \\
\log \left(\sum_{\substack{\mathbf{x}^{[n]} \in \mathbb{X}^{d_{c}} \\
x_{k, m}}} p^{j}\left(y_{n} \mid \mathbf{x}^{[n]}\right) P_{n}^{j}\left(\mathbf{x}^{[n]} \backslash x_{k, m}\right)\right)= \\
\log \left(\sum_{\substack{\left[\mathbf{x}^{[n]} \in \mathbb{X}^{d_{c}} \\
x_{k, m}\right.}} p^{j}\left(y_{n} \mid \mathbf{x}^{[n]}\right) \prod_{\left(k^{\prime}, m^{\prime}\right) \in \mathcal{J}_{n} \backslash(k, m)} P_{n}^{j}\left(x_{k^{\prime}, m^{\prime}}\right)\right) .
\end{gathered}
$$

Where the conditional probability density function (PDF) $p^{j}\left(y_{n} \mid \mathbf{x}^{[n]}\right)$ and a priori probability $P_{n}^{j}\left(x_{k^{\prime}, m^{\prime}}\right)$ are given as

$$
\begin{gathered}
p^{j}\left(y_{n} \mid \mathbf{x}^{[n]}\right) \propto \exp \left(-\frac{1}{\left(2 \sigma^{2}\right)}\left\|y_{n}-\mathbf{h}_{[n]}^{T} \mathbf{x}^{[n]}\right\|^{2}\right), \\
P_{n}^{j}\left(x_{k^{\prime}, m^{\prime}}\right)=\exp \left(L_{n, \rightarrow}^{j}\left(x_{k^{\prime}, m^{\prime}}\right)\right)
\end{gathered}
$$

where $\mathbf{x}^{[n]}$ and $\mathbf{h}_{[n]}$ denote the vector containing the symbols transmitted by every user that spread its data on chip $n$ and their corresponding effective received signature values, respectively. As can be seen in (5) based on received chip $y_{n}$ and a priori input information $P_{n}^{j}\left(x_{k^{\prime}, m^{\prime}}\right)$, extrinsic values are calculated for all the constituent bits involved in (2). Combining (6) and (7) into (5), the message update will be

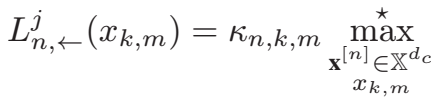

$$
\begin{aligned}
& \left(\sum_{\left(k^{\prime}, m^{\prime}\right) \in \mathcal{J}_{n} \backslash(k, m)} L_{n, \rightarrow}^{j}\left(x_{k^{\prime}, m^{\prime}}\right)-\frac{1}{\left(2 \sigma^{2}\right)}\left\|y_{n}-\mathbf{h}_{[n]}^{T} \mathbf{x}^{[n]}\right\|^{2}\right),
\end{aligned}
$$

where $\kappa_{n, k, m}$ denotes the normalization coefficient and

$$
\max ^{\star}(a, b) \triangleq \log \left(e^{a}+e^{b}\right) .
$$

This technique is termed log-MAP CLi detection. After the message-passing has converged or has reached the maximum number of iterations $J$, a posteriori probability of the transmitted symbol $x_{k, m}$ is estimated as

$$
L_{k, m}\left(x_{k, m}\right)=\sum_{l \in \mathcal{E}_{k, m}} L_{l, \leftarrow}^{J}\left(x_{k, m}\right) .
$$

By using the hard decision, the estimated value of $x_{k, m}$ is

$$
\hat{x}_{k, m}=\arg \max _{x_{k, m} \in \mathbb{X}} L_{k, m}\left(x_{k, m}\right)
$$

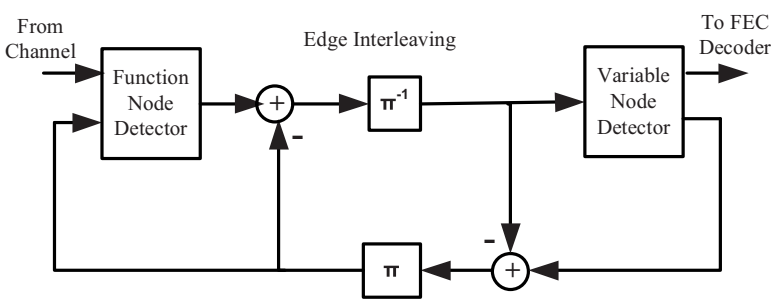

Fig. 2. Iterative MUD for LDS-OFDM.

For a system with forward error correction (FEC), after an appropriate number of iterations the soft output calculated using (10) for each variable node can be sent to the channel decoder to have the final hard decision at the decoder. One of the main advantages of the iterative receiver for LDSOFDM is its ability to support high loads while maintaining the affordable complexity and acceptable performance. In the next section we will show how using EXIT chart analysis helps us improve the performance of the receiver.

\section{EXIT Chart AnAlysis of Multi-User Detector OF LDS SCHEME}

EXIT chart is a useful tool for analysing the flow of information between the constituents of an iterative decoder. This section assesses the extrinsic information transfer characteristics to analyse the transfer of information between the soft-input soft-output (SISO) constituents of the multi-user symbol detector of LDS-OFDM. This would help us find design guidelines to improve the performance of LDS-OFDM.

Although LDS-OFDM by nature is designed for channels that vary over frequency, the design guidelines are related to the performance of the algorithm used for symbol detection. Therefore, the evaluation is carried out for the AWGN channel and then it is extended to the multi-path fading channel. It is important to note that the results presented at this stage are related to a system without FEC.

\section{A. Application of EXIT Charts to the MUD of LDS Scheme}

In order to be able to apply EXIT charts to the MUD of LDS-OFDM we must first explain its structure. The factor graph of LDS receiver contains variable and function nodes, which are connected via appropriate edges. Using MPA in an iterative manner, variable nodes calculate the extrinsic messages related to function nodes according to a priori information they receive from other connected function nodes, and the same rule applies to the extrinsic messages that function nodes send to variable nodes. Note that the message that is exchanged on each edge on either direction only contains information about the corresponding variable node. To be able to evaluate the transformation of extrinsic information for the detector, the sets of variable and function nodes are referred to as variable node detector (VND) and function node detector (FND), respectively. The edge interleaver connects the function nodes and variable nodes, each of which represents a spreading matrix. Fig. 2 shows the structure of the iterative LDS multi-user detector. As it is shown the extrinsic L-values that have been passed on are considered as a priori information 


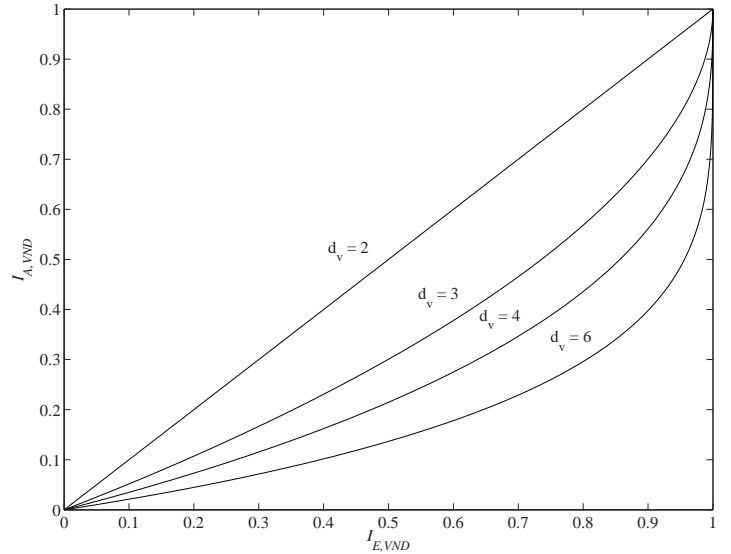

Fig. 3. VND EXIT curves for different $d_{v}$.

by the other detector. In this section, EXIT charts are used to characterize the operation of these two iterative detectors.

EXIT charts are applied to the MUD of LDS using the property of the factor graph of LDS receiver that both variable and function nodes exchange the same information. The information exchanged is the soft value that represents the reliability of the symbol related to each edge. In other words, the information on each edge is about the adjusted variable node, whether it is sent from the variable node itself or the connected function node. Therefore, knowing the transmitted signal we could find the mutual information between the actual value for the variable nodes and the a priori or extrinsic values on the edges. In this paper, $I_{A}$ refers to the average mutual information between the bits on the detector graph edges and the a priori L-values. Also, $I_{E}$ is the average mutual information between the bits on the graph edges and the extrinsic L-values. Therefore, in order to find the EXIT curve for each detector constituent we must represent $I_{E}$ as a function of $I_{A}$ for VND and FND separately.

1) EXIT Curve for the Variable Node Detector: In a regular LDS each variable node has $d_{v}$ incoming messages from the edge interleaver, which is equal to the variable node degree in the factor graph. The variable node produces its messages by calculating (3) for $d_{v}$ connected function nodes. In order to compute an EXIT curve for variable nodes, $L_{l, \leftarrow}^{j-1}\left(x_{k, m}\right)$ in (3) is modelled as the soft output of an AWGN channel when the input is the $l^{\text {th }}$ interleaver bit transmitted using BPSK (binary phase shift keying). Then the mutual information between the variable node's extrinsic messages and actual values of symbols on the edges is calculated. Thus, a priori L-value can be calculated by

$$
A=\mu_{A} x+n_{A}
$$

where $n_{A}$ is an independent Gaussian random variable with variance $\sigma_{A}^{2}$ and mean zero and $x \in \pm 1$ are original bits on the detector graph edges. Then we will have

$$
\mu_{A}=\frac{\sigma_{A}^{2}}{2} .
$$

The mutual information $I_{A}=I(X ; A)$ can be calculated by [11]

$$
\begin{gathered}
I_{A}=\frac{1}{2} \sum_{x=-1,1} \int_{-\infty}^{+\infty} p_{A}(\xi \mid X=x) \\
\quad \log _{2} \frac{2 p_{A}(\xi \mid X=x)}{p_{A}(\xi \mid X=-1)+p_{A}(\xi \mid X=1)} d \xi .
\end{gathered}
$$

Knowing that the conditional probability density function $p_{A}(\xi \mid X=x)$ depends on L-value $A$, with Gaussian distribution and with properties mentioned in (14), we can write

$$
\begin{aligned}
& I_{A}\left(\sigma_{A}\right)=1- \\
& \quad \int_{-\infty}^{+\infty} \frac{e^{-\left(\left(\xi-\sigma_{A}^{2} / 2\right)^{2} / 2 \sigma_{A}^{2}\right)}}{\sqrt{2 \pi} \sigma_{A}} \log _{2}\left(1+e^{-\xi}\right) d \xi .
\end{aligned}
$$

For abbreviation we define

$$
J(\sigma):=I_{A}\left(\sigma_{A}=\sigma\right),
$$

with

$$
\lim _{\sigma \rightarrow 0} J(\sigma)=0, \quad \lim _{\sigma \rightarrow \infty} J(\sigma)=1, \quad \sigma \geq 0 .
$$

Considering (3) together with the fact that the sum of two normally distributed random variables is also normally distributed with the mean and variance equal to the sum of theirs, the EXIT function of a degree $d_{v}$ variable node is

$$
I_{E, V N D}\left(I_{A}, d_{v}\right)=J\left(\sqrt{\left(d_{v}-1\right)\left(J^{-1}\left(I_{A}\right)\right)^{2}}\right) .
$$

Fig. 3 plots variable node EXIT curves for different $d_{v}$ s using (18).

2) EXIT Curve for the Function Node Detector: A function node of degree $d_{c}$ has $d_{c}+1$ incoming messages, $d_{c}$ from the edge interleaver and one message from the channel. The output L-values are calculated in (8) in details. We model a priori Lvalues, $L_{n, \rightarrow}^{j}\left(x_{k^{\prime}, m^{\prime}}\right)$, as the output of an AWGN channel that its input is the corresponding transmitted bit using BPSK and then calculate the mutual information of output with regards to the actual value on the edges. Due to the complexity of the calculation in function nodes, the EXIT curve for them is computed by simulations over AWGN channel. Therefore, first the distribution $p_{E}$ for extrinsic information is determined by Monte Carlo simulation (histogram measurements). Then using (14) the mutual information between the extrinsic information and the bits on the detector graph edges, is calculated.

Fig. 4 illustrates the EXIT charts for the MUD with 252 chips and different number of users (overloading conditions) which lead to different $d_{c}$ s for a fixed $d_{v}=2$. Fig.4 shows that for a load of $400 \%$ the performance degrades noticeably compared to a load of $200 \%$ and $300 \%$. As we can see in this figure, the iterative process starts with $I_{A, F N D}=0$ since no prior information is given to the function nodes in the beginning. In the next steps, the output L-values are exchanged between VND and FND curves. Fig. 4 also shows that the FND curves for different loading conditions intersect at $I_{A}=1$, which is because for complete a priori information, the function nodes are able to eliminate the multiple access 


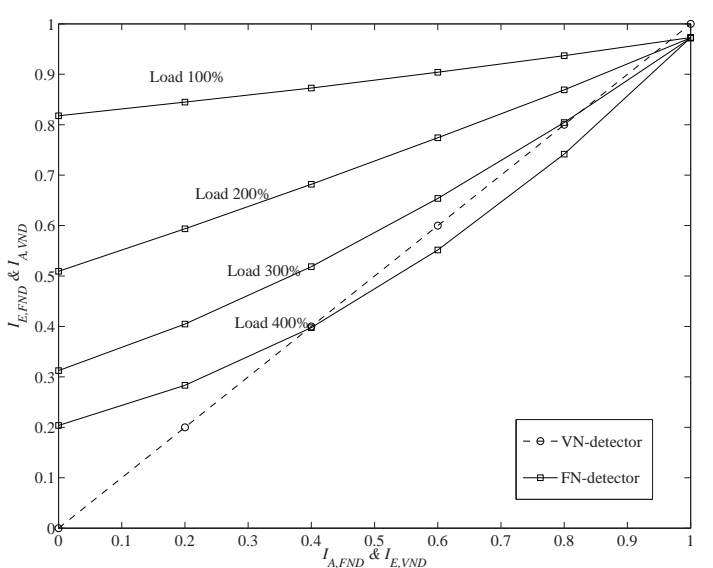

Fig. 4. EXIT charts for MUD at $E_{b} / N_{0}=8 \mathrm{~dB}$ for different $d_{c}$ s.

interference (MAI) fully and the effect of overloading will be cancelled. Fig. 5 reveals the EXIT charts and the trajectories of the system for $E_{b} / N_{0}$ equal to $5 \mathrm{~dB}$ and $8 \mathrm{~dB}$. It shows that an increase in signal to noise ratio (SNR) would shift the FND curve vertically towards higher extrinsic output. This figure also shows the trajectories of the iterative process obtained by simulation. Except for a slight difference in the early iterations, which occurs because of the finite size of edge interleaver in the LDS structure, the system trajectories closely follow the transfer curves of the MUD components, which indicates that the EXIT charts analysis is valid for LDS-OFDM's MUD.

\section{B. EXIT Chart Analysis Aided Signature Design for LDS}

There are different parameters in the design of the LDS matrix that affect the performance of the system. Two important parameters are the number of chips that each symbol is spread on, $d_{v}$, and the number of symbols that share the same chip, $d_{c}$. The relation between these two parameters for overloading equal to $\beta$ is as follows:

$$
d_{c}=\beta d_{v} .
$$

To design a robust LDS, the EXIT charts are drawn for different $d_{v}$ s to evaluate the effect of processing gain on the convergence behavior of MUD. Fig. 6 shows the EXIT charts for a load of $200 \%$ and $E_{b} / N_{0}=8$ for an LDS design with different processing gains. As it can be noted, using $d_{v}=3$ improves the intersection point compared to $d_{v}=2$. This is due to the larger frequency diversity offered at $d_{v}=3$. Also the performance at $d_{v}=4$ is close to $d_{v}=3$, therefore considering that complexity increases exponentially with $d_{v}$ (for fixed overloading conditions), $d_{v}=3$ can be regarded as a suitable value in the design of LDS systems with a load of $200 \%$. Thus, as an example, we have found that $d_{v}=3$ is a proper value for a load of $200 \%$, this can be extended to other scenarios to find suitable parameters for them.

\section{EXIT Chart Analysis of Turbo Multi-User DETECTOR/DECODER}

In this section we propose a new receiver for LDS-OFDM based on iterative exchange of extrinsic information between

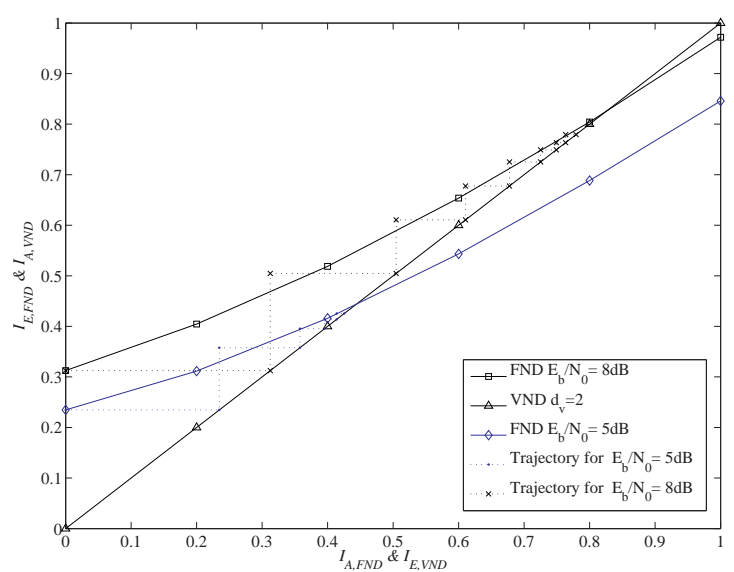

Fig. 5. EXIT chart for MUD at $E_{b} / N_{0}=8 \& 5 \mathrm{~dB}$ for load $200 \%$.

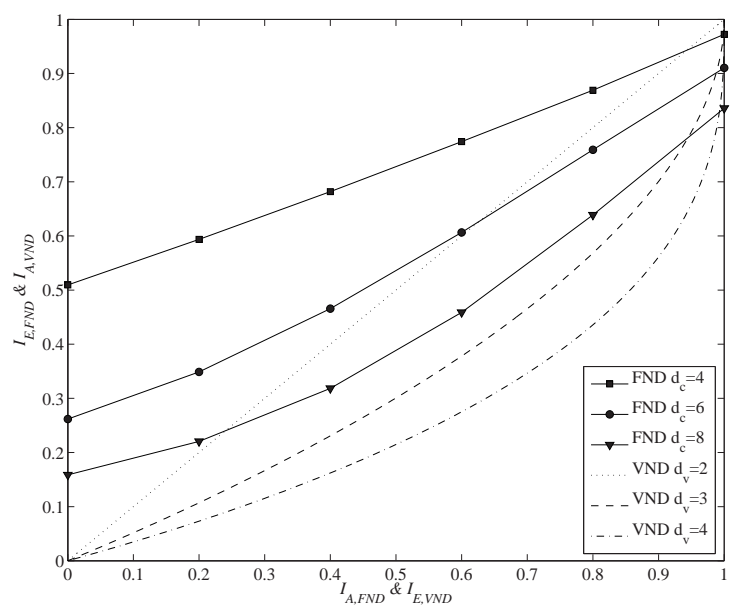

Fig. 6. EXIT chart for MUD at $E_{b} / N_{0}=8 \mathrm{~dB}$ for different $d_{c} \& d_{v}$.

LDS multi-user detector and FEC decoders of different users. There exist two iterative processes in the turbo MUDD: inner processing for MUD and outer processing for turbo MUDD. Having two iterative processes involved, the message update algorithm must be modified accordingly. Defining $t$ as the turbo iteration index for outer iteration, the message updates in (3) and (4) can be adapted

$$
\begin{aligned}
& L_{n, \rightarrow}^{j, t}\left(x_{k, m}\right)=\sum_{l \in \mathcal{E}_{k, m} \backslash n} L_{l, \leftarrow}^{j-1, t}\left(x_{k, m}\right)+L_{\text {dec }, \text { out }}^{t-1}\left(x_{k, m}\right), \\
& L_{n, \leftarrow}^{j}\left(x_{k, m}\right) \propto \\
& f\left(x_{k, m} \mid y_{n}, L_{n, \rightarrow}^{j, t}\left(x_{k^{\prime}, m^{\prime}}\right), \forall\left(k^{\prime}, m^{\prime}\right) \in \mathcal{J}_{n} \backslash(k, m)\right) .
\end{aligned}
$$

Where $L_{\text {dec,out }}^{t-1}$ is the extrinsic information that an FEC decoder sent to its corresponding variable node in the prior turbo iteration. Therefore, considering the message-passing algorithm the messages sent by variable nodes to the decoder are as follows:

$$
L_{d e c, i n}^{j, t}=\sum_{l \in \mathcal{E}_{k, m}} L_{l, \leftarrow}^{j-1, t}\left(x_{k, m}\right) .
$$




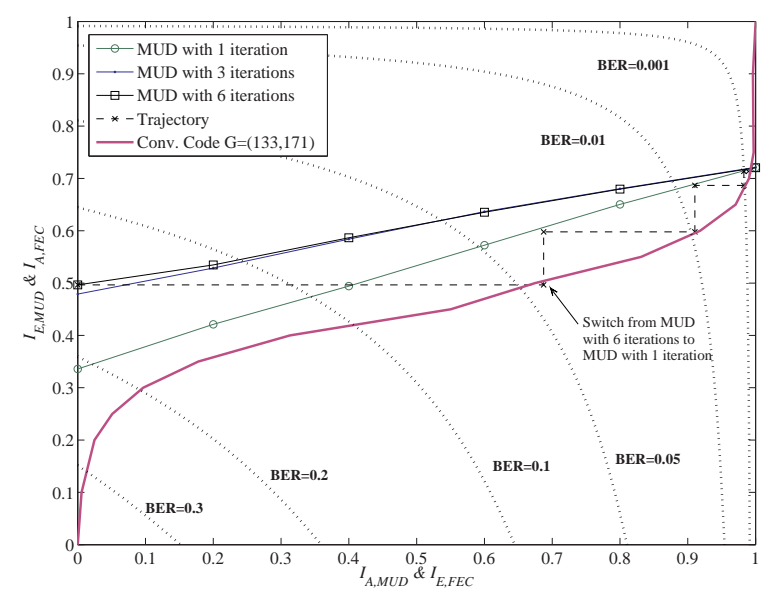

Fig. 7. EXIT charts for turbo MUDD at $E_{b} / N_{0}=0.5 \mathrm{~dB}$ for load $200 \%$.

Applying the message-passing rule to the new graph, the message sent by the variable node to a function node must be based on all the messages it has received from the remainder of the connected function nodes in addition to the message that has been received from the corresponding FEC decoder. For function nodes the message update follows the same procedure as for the conventional LDS-OFDM MUD.

In this section, EXIT charts are used to investigate and reduce the complexity of the turbo MUDD. To calculate the EXIT charts, both the MUD and FEC decoder are assumed to be a device that produce a new sequence of extrinsic information using a sequence of observations and the input $a$ priori information. Therefore, in order to calculate the mutual information using (15), it is necessary to estimate the PDF from the histogram of the soft output of a constituent decoder. Furthermore, EXIT chart is a useful tool for predicting the BER performance [12]. The BER can be predicted by estimating the soft output of the coded bits by summing up the extrinsic information and the a priori information

$$
\begin{gathered}
\Lambda=L_{M U D}+L_{D E C}, \\
\sigma_{\Lambda}^{2}=\sigma_{L_{M U D}}^{2}+\sigma_{L_{D E C}}^{2},
\end{gathered}
$$

where (24) assumes a priori information and the extrinsic information are independent. Both variances can be calculated from their related mutual information using (17). Thus, the BER can be calculated as follows:

$$
p_{b} \approx \frac{1}{2} \operatorname{erfc}\left(\sqrt{\frac{\mu_{\Lambda}^{2}}{\sigma_{\Lambda}^{2}}}\right)=\frac{1}{2} \operatorname{erfc}\left(\frac{\sigma_{\Lambda}}{2}\right) .
$$

Fig. 7 illustrates the EXIT chart for turbo MUDD with different number of inner iterations for a system load of $200 \%$ over AWGN channel. Here we implemented the system with 60 chips and a convolutional code with generator $G=(133,171)$ in octal notation (FEC decoder). Additionally, the BER scaling according to (25) is given as a contour plot.

Fig. 7 shows that the curves for different numbers of inner iterations intersect at the same point at input mutual information equal to one. This is due to full interference

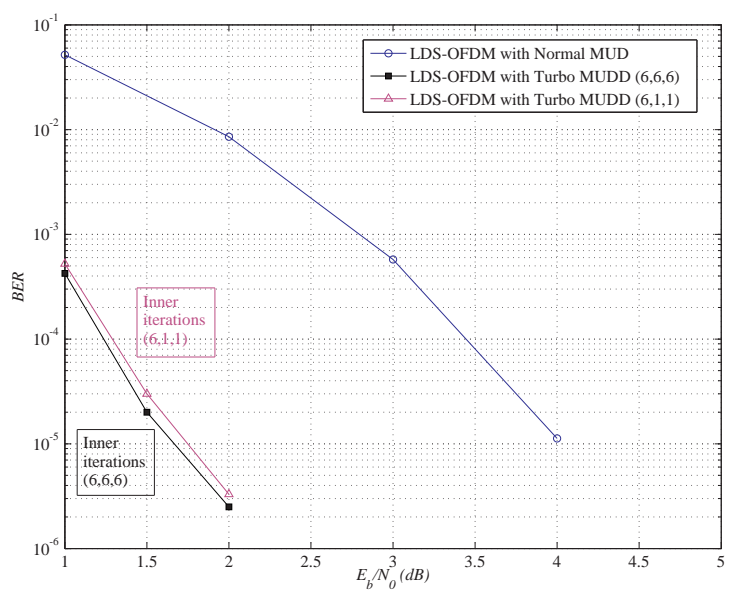

Fig. 8. BER comparison for LDS-OFDM with different MUD techniques.

cancellation at FNDs when perfect a priori information is provided to them. In this case just one inner iteration will suffice to detect the data symbols. However, as it is observed from Fig. 7 larger number of turbo iterations will be required if only one inner iteration is used. In contrast, the cases with 3 and 6 inner iterations exhibits a marginally open tunnel between the EXIT curves for the FEC decoders and the MUD, hence for these cases turbo MUDD can approach its final performance by less number of turbo iterations.

To efficiently utilize both inner and outer iterations we suggest using 6 inner iterations at first turbo iteration and then reducing the inner iterations to 1 from the second turbo iteration and onward. This will considerably reduce the overall complexity while maintaining the same performance. As the simulated trajectory in Fig. 7 shows, the simulated results follow the suggested iteration schedule very closely and the turbo MUDD converges by only 4 turbo iterations.

Monte Carlo based simulation results for LDS-OFDM's BER with a load of $200 \%, N_{c}=60$ over AWGN channel is shown in Fig. 8. This result proves the prediction concluded using EXIT charts. As it is observed, the receiver brings about a significant performance improvement over conventional LDS-OFDM receivers. $2.3 \mathrm{~dB}$ gain at $\mathrm{BER}=10^{-5}$ is achieved by turbo MUDD with 3 turbo iterations over conventional LDS-OFDM receiver. As expected from EXIT chart analysis, the performance of the turbo MUDD with $(6,1,1)$ inner iterations is close to the one with $(6,6,6)$ inner iterations. Consequently, the complexity was reduced while keeping the performance of the two systems close to each other. This result is important because in a real system, turbo iteration requires a huge processing overhead.

Finally, to show the validity of using previous analysis on a fading channel, the EXIT chart is calculated for the turbo LDS-OFDM under multi-path fading channel (ITU Pedestrian Channel B). Fig.9 shows the convergence behavior of LDSOFDM with effective spreading size of 3 and 60 chips and 120 data symbols which belong to 10 users. The graphs for different SNRs show that an increase in the SNR only result in a vertical shift of MUD curve, which is a complete match 
TABLE I

Simulation PARAMETERS

\begin{tabular}{|c|c|c|}
\hline \multicolumn{2}{|c|}{ Number of Users } & 10 \\
\hline \multicolumn{2}{|c|}{ Number of data sub-channels } & 60 \\
\hline \multicolumn{2}{|c|}{ FFT size } & 64 \\
\hline \multicolumn{2}{|c|}{ Sub-channel bandwidth } & $15 \mathrm{KHz}$ \\
\hline \multicolumn{2}{|c|}{ Multipath channel model } & ITU Pedestrian Channel B \\
\hline \multicolumn{2}{|c|}{ LDS-OFDM MUD technique } & Max-Log-MAP \\
\hline \multicolumn{2}{|c|}{ Channel coding } & Half-rate convolutional code \\
\hline \multicolumn{2}{|c|}{ Modulation } & BPSK \\
\hline \multirow{2}{*}{ Data streams per user } & $100 \%$ Loading & 6 \\
\hline & $200 \%$ Loading & 12 \\
\hline \multicolumn{2}{|c|}{ Effective spreading factor (LDS) } & $d_{v}=3$ \\
\hline \multirow{2}{*}{$\begin{array}{l}\text { Symbols per } \\
\text { sub-carrier (LDS) }\end{array}$} & $100 \%$ Loading & $d_{c}=3$ \\
\hline & 200\% Loading & $d_{c}=6$ \\
\hline
\end{tabular}

to the behaviour of the system for AWGN channel shown in Fig. 5. It is also shown that the simulated decoding trajectory travel within the open tunnel between the EXIT curves for the MUD and the FEC decoder. Therefore, the validity of EXIT chart analysis for multi-path fading channels can be verified. It is necessary to mention that EXIT chart analysis assumes that the PDF of the exchanged messages approaches Gaussianlike distributions with increasing number of iterations, thus, it can be applied under multi-path fading channel as long as the trajectory follows the curves of the receiver components.

The receiver that its parameters are selected according to our proposed design guidelines can be called tailor-designed LDS receiver. In next section, the performance of a tailordesigned LDS-OFDM system is shown and compared with well-known multiple access techniques with similar principles.

\section{PERFormanCE COMPARISON WITH MC-CDMA AND GO-MC-CDMA}

In this section, the performance of tailor-designed LDSOFDM is evaluated and compared with MC-CDMA and GOMC-CDMA in terms of BER performance and computational complexity. Considering the prohibitive complexity of the optimum MUD, LDS-OFDM is compared with a MC-CDMA system that has linear minimum mean-square error (MMSE) detector. The linear MMSE detector is the optimal linear detector that maximizes the output signal-to-interference and noise ratio (SINR) [13]. On the other hand, it has been shown that the performance of GO-MC-CDMA is very close to the single-user bound [6], thus it is necessary to show that the proposed receiver design for LDS-OFDM is able to keep the performance the same while reducing the complexity.

The performances are evaluated using Monte Carlo based simulations over multi-path fading channel. The single-user BER bound with the same channel profile is also considered in the comparison. Throughout the simulations, the overall number of information bits transmitted by all the systems is kept equal to ensure a fair comparison between the systems. The simulation parameters are listed in Table I. The number of sub-carriers used is the same as in [6] to keep the complexity

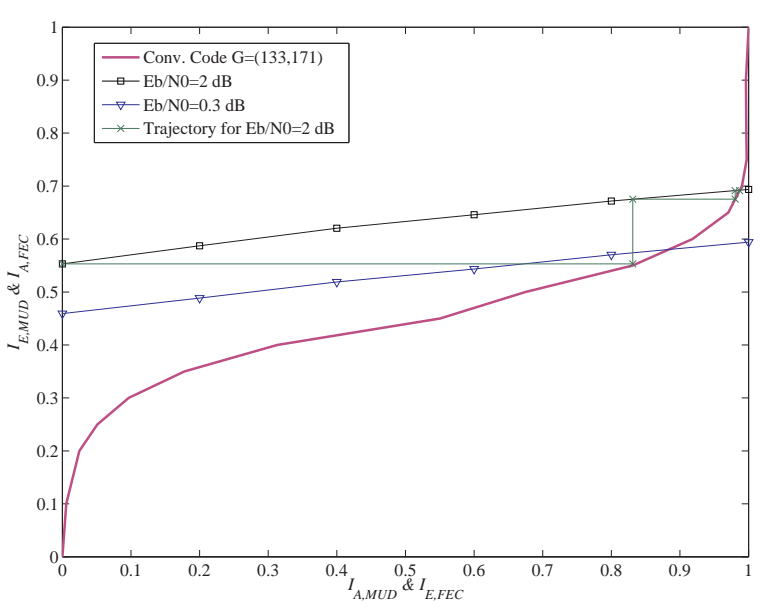

Fig. 9. EXIT chart for turbo MUDD for $200 \%$ load under multi-path fading channel.

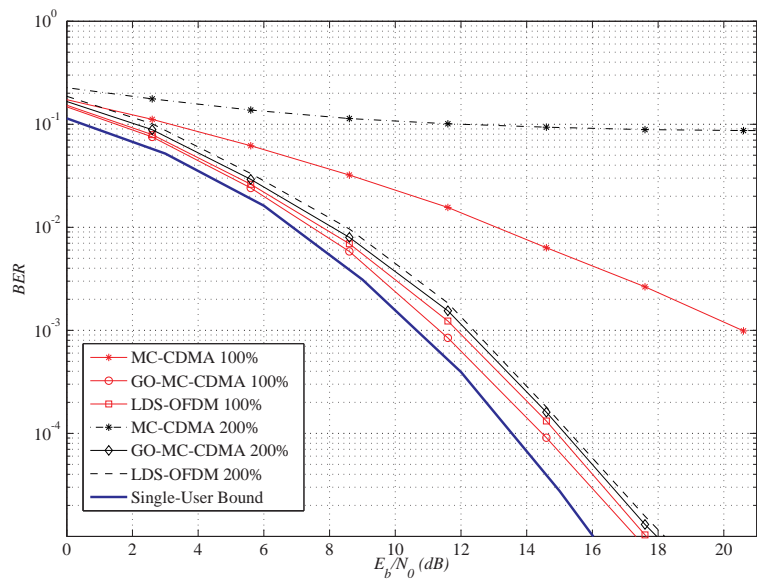

Fig. 10. Performance results for LDS-OFDM, MC-CDMA and GO-MCCDMA.

of the optimum MUD feasible. For GO-MC-CDMA, the number of sub-carriers per group is set to 4 and maximum likelihood (ML) detection is employed per group. For MCCDMA the processing gain is equal to the number of data sub-channels. For MC-CDMA and GO-MC-CDMA systems orthogonal codes for a load of $100 \%$ and Welch bound equality (WBE) for a load of $200 \%$ are used. The spreading codes are constructed using the algorithm developed in [14]. A regular graph structure is maintained for LDS-OFDM while its signatures are generated randomly. Using the design guidelines achieved in section III, the effective spreading factor for LDSOFDM is equal to 3 . To keep the complexity of LDS-OFDM's receiver at a similar level with the receiver for MC-CDMA and GO-MC-CDMA, its receiver is not turbo MUDD (the performance of turbo MUDD is shown in section IV).

Fig. 10 shows the BER results for systems with loads of $100 \%$ and $200 \%$. As it can be observed from the figure, LDSOFDM system achieves performance close to the GO-MCCDMA system, which employs optimum MUD. However, the complexity of the LDS detector is less than the complexity of GO-MC-CDMA. As in LDS-OFDM the complexity increases 
exponentially by the number of symbols per sub-carrier $\left(d_{c}\right)$, a complexity order of $\mathcal{O}\left(|\mathbb{X}|^{3}\right.$ ) and $\mathcal{O}\left(|\mathbb{X}|^{6}\right)$ is required for a load of $100 \%$ and $200 \%$, respectively. However, in GOMC-CDMA the complexity increases exponentially by the number of transmitted symbols in each group, which results in complexity order of $\mathcal{O}\left(|\mathbb{X}|^{4}\right)$ and $\mathcal{O}\left(|\mathbb{X}|^{8}\right)$ for loads of $100 \%$ and $200 \%$, respectively. Furthermore, the results show that, LDS-OFDM outperforms the performance of MC-CDMA with MMSE detector. This is because the LDS detector is more efficient than the MMSE detector in eliminating the MUI. For a load of $100 \%$, LDS-OFDM outperforms MC-CDMA by $8.7 \mathrm{~dB}$ at $\mathrm{BER}=10^{-3}$. The MMSE detector fails to attain a satisfactory BER performance under the overloaded conditions. Considering all the results, it can be concluded that the tailor-designed LDS-OFDM system can achieve performance close to the GO-MC-CDMA system with less complexity. Moreover, the LDS-OFDM system outperforms the system that employs MC-CDMA with linear MMSE detector. At the end, to highlight the importance of LDS-OFDM receiver we will compare this newly proposed technique with the existing multiple access techniques in next section.

\section{LDS-OFDM IN COMPARISON WITH EXISTING Multiple ACCESS TeChNiques}

In this section, the properties of LDS-OFDM are discussed and the scheme is compared with different MAC techniques.

\section{A. Frequency Diversity}

For OFDMA systems, it is not possible to exploit the frequency domain diversity at modulation symbol level because the symbols of users are assigned directly to sub-channels in such systems. Therefore, in order to exploit the frequency diversity for OFDMA system at a later stage, it is crucial to incorporate properly the designed error correction coding and interleaving schemes [15].

For LDS-OFDM, the performance of the system was improved by introducing a larger degree of frequency diversity proportional to $d_{v}$, the effective spreading factor. Furthermore, considering that the detection of user symbols in LDS structure is done completely in the sub-carrier level, frequency diversity can be achieved by assigning distributed and spaced-sufficient sub-carriers for spreading of a given data symbol. Also, the created LDS structure will produce diversity on the interference experienced by each data symbol. Although LDS-OFDM better exploits frequency diversity, as compared to OFDMA, its diversity gain is less than the one for MC-CDMA and GOMC-CDMA, as they offer full frequency diversity [6], [16].

\section{B. Computational Complexity of MUD}

Applying the close-to-optimum MUD based on MPA became feasible for LDS structure by reducing the number of interferers in each chip. As mentioned earlier, $d_{c}$ is the number of symbols that interfere with each other on each chip. Therefore, at each received chip a user's symbol will have only $d_{c}-1$ interferers, where $d_{c} \ll K$.

The complexity of the proposed receiver for LDS-OFDM will be in order of $\mathcal{O}\left(|\mathbb{X}|^{d_{c}}\right)$ which is a considerable reduction compared to $\mathcal{O}\left(|\mathbb{X}|^{K}\right)$ for optimal MUD. The complexity of LDS MUD will be

$$
\text { Complexity }=|\mathbb{X}|^{d_{c}} \times(\text { No. of MUD iterations }) \times N_{c} \text {. }
$$

Consequently, although the complexity is increased compared to OFDMA, its growth is much less than the optimum MUD used in MC-CDMA. Comparing the factor graph of GO-MC-CDMA and LDS-OFDM we can see that in contrast to the LDS structure for GO-MC-CDMA in each group there is full connection between the function nodes and variable nodes and there is no connection between the groups. Therefore, applying message-passing algorithm is inefficient due to the full connectivity of the graph for GO-MC-CDMA. For GO-MC-CDMA the complexity of the system depends on the size of orthogonal groups because the complexity for maximum likelihood detection in GO-MC-CDMA increases exponentially with the number of active users per group [6]. As mentioned earlier the complexity of LDS-OFDM is less than the complexity of GO-MC-CDMA.

\section{Overloaded Conditions}

In wireless systems the number of users naturally exceeds the available dimensions as the demand for the spectrum is increasing while the bandwidth is limited. Conventional signature design for multiple access systems such as MC-CDMA and GO-MC-CDMA is based on orthogonality between the sequences to avoid interference. Therefore, these systems can perform reliably when the number of users is equal to the number of chips [17]. However, overloaded conditions can be supported by LDS-OFDM as the design of this system is not based on orthogonality. The LDS system is able to approach near single-user performance for a CDMA system with up to $200 \%$ loading [18].

\section{Near-Far Effect}

The near-far problem (i.e., the effect of unequal received energies) is the principal shortcoming of direct sequence CDMA systems. On the other hand, by jointly detecting all users' symbols, optimum multi-user detection for CDMA systems is near-far resistant [19]. Considering that in the MUD of LDS-OFDM an iterative joint process has been employed for detecting different users' symbols and the fact that the performance of LDS MUD is close to optimum MUD, we can conclude that LDS-OFDM is robust against unequal received powers. Fig. 11 shows the performance of near-far resistance for LDS-OFDM MUD with different numbers of chips. The simulation is done for the case where $E_{b} / N_{0}$ is equal to 15 $\mathrm{dB}$ for the first user, and $E_{b} / N_{0}$ of other users is different. The BER performance of the first user is shown according to $\Delta E_{b} / N_{0}$, which represents the difference in $E_{b} / N_{0}$ of user of interest from the $E_{b} / N_{0}$ of other users (all other users have equal $E_{b} / N_{0}$ ). The results are shown for an LDSOFDM system with different numbers of chips and loads. It can be seen that unequal received power has a minor effect on the performance of user of interest for all the scenarios. Furthermore, the performance of the LDS-CDMA system against the near-far effect problem has been analysed in [18]. 


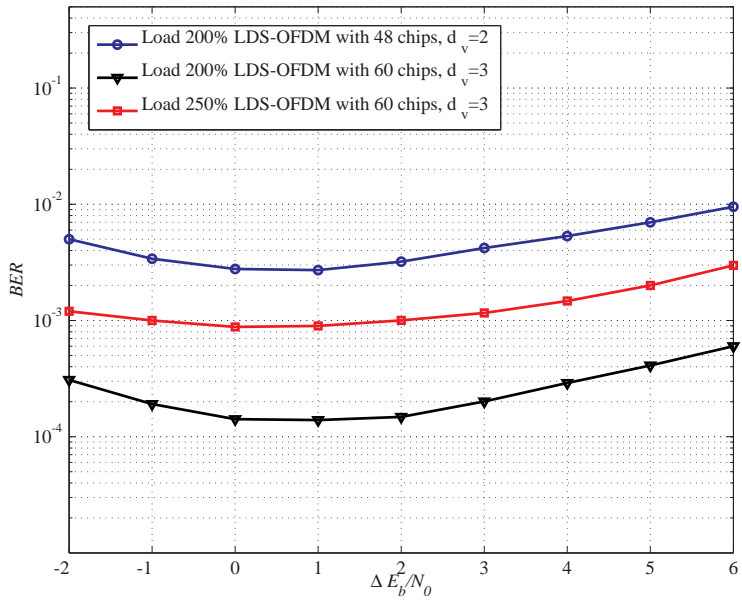

Fig. 11. Near-far resistance performance of LDS-OFDM.

These results match the results shown for LDS-OFDM, since both techniques are based on low density signatures. In order to achieve an even better near-far resistance, the system can resort to power control mechanisms. Several algorithms are introduced in the literature for the optimum power control of MC-CDMA systems [20]-[22], which can be applied to LDSOFDM if necessary.

\section{CONCLUSIONS}

In this paper, a close-to-optimum MUD for LDS-OFDM systems was introduced and analysed. The LDS-OFDM receiver was evaluated using EXIT charts. By analyzing the inner iterations for MUD under AWGN channel, it was shown that the signature design with effective processing gain equal to 3 was a suitable value for a load of $200 \%$, considering the complexity and performance. This could be extended to other scenarios in order to find suitable values for other conditions. In addition, we showed how the performance is affected by loading as the curves intersected in lower mutual information points for higher loading values. Furthermore, the proposed turbo MUDD was investigated and by deriving and analysing its EXIT charts we could reduce the complexity by properly tuning the number of inner iterations along the outer turbo iterations. It was shown that $2.3 \mathrm{~dB}$ performance improvement at BER equal to $10^{-5}$ could be obtained when turbo MUDD was employed over an AWGN channel.

Applying the achieved guidelines, the performance of LDSOFDM was evaluated over a typical multi-path fading channel under different spectral efficiency conditions. The simulation results showed a noticeable performance improvement compared to MC-CDMA systems with MMSE MUD. This large performance gain could be achieved at the cost of slightly increased computational complexity. This improvement is mainly due to capability of LDS-OFDM to exploit frequency domain diversity in addition to avoiding a strong interference to corrupt all the sub-carriers. In terms of complexity it was shown that the performance of LDS-OFDM was close to GO-MC-CDMA while reducing the computational complexity of the MUD. Interference handling property of LDS-OFDM makes it a suitable candidate for use in heterogeneous wireless networks. However, their use in these specific scenarios needs further detailed analysis.

\section{ACKNOWLEDGMENT}

This work was supported by Huawei Technologies Co., Ltd, China. The authors would also like to thank Colin O'Reilly for his editorial comments.

\section{REFERENCES}

[1] N. Yee and J.-P. Linnartz, "BER of multi-carrier CDMA in an indoor Rician fading channel," in Proc. Conf Signals, Systems and Computers Record of The Twenty-Seventh Asilomar Conf, 1993, pp. 426-430.

[2] K. Fazel, "Performance of CDMA/OFDM for mobile communication system," in Proc. 2nd Int Conf. on Universal Personal Communications: Gateway to the 21st Century, vol. 2, 1993, pp. 975-979.

[3] A. Chouly, A. Brajal, and S. Jourdan, "Orthogonal multicarrier techniques applied to direct sequence spread spectrum CDMA systems," in Proc. IEEE Global Telecommunications Conf. (GLOBECOM '93), 1993, pp. 1723-1728.

[4] A. Persson, T. Ottosson, and E. Strom, "Time-frequency localized CDMA for downlink multi-carrier systems," in Proc. IEEE Seventh Int Spread Spectrum Techniques and Applications Symp, vol. 1, 2002, pp. $118-122$.

[5] S. Verdu, Multiuser Detection. Cambridge University Press, 1998.

[6] C. Xiaodong, Z. Shengli, and G. B. Giannakis, "Group-orthogonal multicarrier CDMA," IEEE Trans. Commun., vol. 52, no. 1, pp. 9099, 2004.

[7] R. Hoshyar, R. Razavi, and M. Al-Imari, "LDS-OFDM an efficient multiple access technique," in Proc. IEEE 71st Vehicular Technology Conf. (VTC 2010-Spring), 2010, pp. 1-5.

[8] J. Choi, "Low density spreading for multicarrier systems," in Proc. IEEE Eighth Int Spread Spectrum Techniques and Applications Symp, 2004, pp. 575-578.

[9] F. R. Kschischang and B. J. Frey, "Iterative decoding of compound codes by probability propagation in graphical models," IEEE J. Sel. Areas Commun., vol. 16, no. 2, pp. 219-230, 1998.

[10] R. Razavi, M. Ali Imran, and R. Tafazolli, "EXIT chart analysis for turbo LDS-OFDM receivers," in Proc. 7th Int. Wireless Communications and Mobile Computing Conf. (IWCMC), 2011, pp. 354-358.

[11] S. Ten Brink, "Convergence behavior of iteratively decoded parallel concatenated codes," IEEE Trans. Commun., vol. 49, no. 10, pp. 17271737, 2001.

[12] L. Kai and W. Xiaodong, "EXIT chart analysis of turbo multiuser detection," IEEE Trans. Wireless Commun., vol. 4, no. 1, pp. 300-311, 2005.

[13] D. N. C. Tse and S. V. Hanly, "Linear multiuser receivers: effective interference, effective bandwidth and user capacity," IEEE Trans. Inf. Theory, vol. 45, no. 2, pp. 641-657, 1999.

[14] S. Ulukus and R. D. Yates, "Iterative construction of optimum signature sequence sets in synchronous CDMA systems," IEEE Trans. Inf. Theory, vol. 47, no. 5, pp. 1989-1998, 2001.

[15] D. Tse and P. Viswanath, Fundamentals of Wireless Communication. Cambridge, 2005.

[16] S. Zhou, G. B. Giannakis, and A. Swami, "Frequency-hopped generalized MC-CDMA for multipath and interference suppression," in Proc. 21st Century Military Communications MILCOM 2000, vol. 2, 2000, pp. 937-941.

[17] M. M. B. J. Choi T. Keller L. Hanzo, OFDM and MC-CDMA for Broadband Multi-User Communications, WLANs and Broadcasting. John Wiley \& Sons Ltd, 2003.

[18] R. Hoshyar, F. P. Wathan, and R. Tafazolli, "Novel low-density signature for synchronous CDMA systems over AWGN channel," IEEE Trans. Signal Process., vol. 56, no. 4, pp. 1616-1626, 2008.

[19] X. Yue and H. H. Fan, "Nearfar resistance of optimum and suboptimum CDMA detectors under multipath," IEEE Trans. Signal Process., vol. 53, pp. 2898-2917, 2005.

[20] S. P. W. Jarot and M. Nakagawa, "Transmission power control techniques for the reverse link of OFDM-DS-CDMA system," in Proc. IEEE Int Computers and Communications Symp, 1999, pp. 331-337. 
[21] J. G. Andrews and T. H. Y. Meng, "Performance of multicarrier CDMA with successive interference cancellation in a multipath fading channel," IEEE Trans. Commun., vol. 52, no. 5, pp. 811-822, 2004.

[22] A. Hamid, R. Hoshyar, and R. Tafazolli, "Band based power control (BBPC) for MC-CDMA radio interface," in Proc. VTC2004-Fall Vehicular Technology Conf. 2004 IEEE 60th, vol. 1, 2004, pp. 404-408.

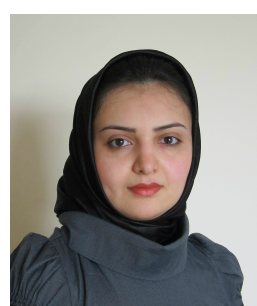

Razieh Razavi (S'10) received her B.Sc. degree in electrical engineering from University of Tehran, Tehran, Iran, and her M.Sc. degree (with distinction) in Wireless Communication Systems from Brunel University, London, United Kingdom, in 2006 and 2008 , respectively. She is currently working toward the Ph.D. degree in mobile communications in the Centre for Communication Systems Research (CCSR), University of Surrey, Surrey, U.K.

Her research interests include information theory, advanced multi-user detection and decoding techniques, advanced multiple access techniques, and stochastic processes.

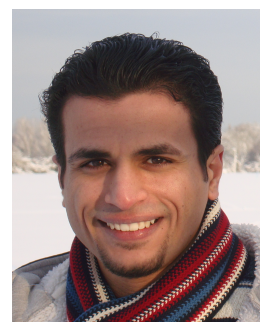

Mohammed Al-Imari (S'10) received the B.Sc. degree in electronic and communications engineering from University of Technology, Baghdad, Iraq, in 2005, and the M.Sc. degree (with distinction) in wireless communication systems from Brunel University, London, UK, in 2008. He secured first rank in his B.Sc, and M.Sc, degrees along with the IEEE Communications Prize for the best project from the department of Electronic and Computer Engineering, Brunel University, for the 2008/2009 academic year. He is currently pursuing the Ph.D. degree in mobile communications at the University of Surrey, U.K. His research interests include information theory, multiple access techniques, and radio resource management in multicarrier communications.

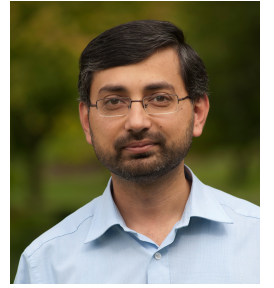

Dr. Muhammad Ali Imran (M'03) received his M.Sc. (Distinction) and Ph.D. degrees from Imperial College London, UK, in 2002 and 2007, respectively. He secured first rank in his B.Sc. and a distinction in his M.Sc. degree along with an award of excellence conferred by the President of Pakistan, in recognition of his academic achievements. He is currently a lecturer in the Centre for Communication Systems Research (CCSR) at the University of Surrey, UK. He has been actively involved in European Commission funded research projects ROCKET and EARTH, Mobile VCE funded project on fundamental capacity limits and EPSRC-UK funded project India-UK ATC. For EARTH project he coordinated CCSR research theme. For IU-ATC project he lead the research theme on self organising networks. He is the principal investigator of EPSRC-UK funded multidisciplinary REDUCE project aiming at using ICT to reduce energy consumption of users. He is also involved in several industry funded projects on the design of spectrum and energy efficient link and system level solutions for wireless communications. His main research interests include the analysis and modelling of the physical layer, optimisation for the energy efficient wireless communication networks and the evaluation of the fundamental capacity limits of wireless networks.

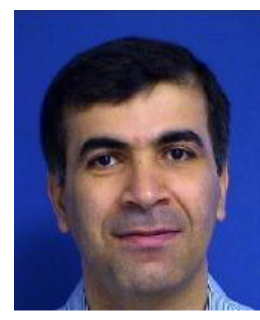

Reza Hoshyar received his B.S. degree in communications engineering and his M.S. and Ph.D. degrees, both in mobile communications, from Tehran University, Tehran, Iran, in 1991, 1996, and 2001, respectively. He received the top and second rank awards for his B.S. and M.S. degrees from Tehran University. From 2002 to September 2010, he has been a Research Fellow and then Senior Research Fellow in the Mobile Communications Research Group, Centre for Communication Systems Research (CCSR), University of Surrey, Surrey, United Kingdom. During this period, he was actively involved in several European projects focusing on advanced digital signal processing, coding and modulation, and scheduling techniques for relaying, and cooperative communications in OFDM/OFDMA systems. Currently he is a principal system engineer at Silicon Valley Labs, Texas Instruments, California, USA. His current focus is on advanced mixed analog/digital signal processing and compressive sensing.

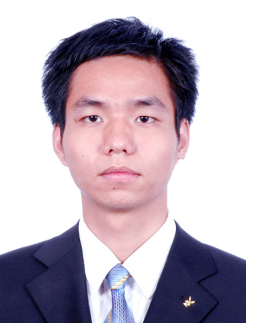

Dageng Chen (S'04, M'06) was born in Hubei, China in 1982. He received the B.E. degree in 2004 and the M.S. degree in 2006, both from the Wuhan University at Wuhan.

$\mathrm{He}$ is currently one of the team leaders in Communication Technologies Lab, Huawei, Shanghai. His primary research focus is on advanced wireless communications, next generation communication system, large scale cooperation system, link and system level evaluation. 